\title{
Abordaje quirúrgico y evolución posoperatoria en reintervenciones de complicaciones en cirugía colorrectal laparoscópica, 15 años de experiencia
}

\author{
Sebastián Olivares M. ${ }^{1,2}$, Marcelo Rodríguez G. ${ }^{1,2}$, \\ Alejandro Readi V. ${ }^{1,2}$ y Gonzalo Campaña V.,
}

\section{Surgical approach and postoperative clinical course in reinterventions for complications following laparoscopic colorectal surgery: 15 years of experience}

\begin{abstract}
Aim: To compare three approaches (laparoscopic, open, and conversion of laparoscopic approach) for the management of intra-abdominal surgical complications after elective laparoscopic colorectal surgery. Materials and Method: This was a retrospective cohort study including patients who required reoperation due to an intra-abdominal surgical complication after initial elective laparoscopic colorectal surgery. Patients were grouped according to the reoperation approach-laparoscopic reoperation, laparoscopic reoperation that required conversion to open surgery, and open reoperation. Pre-operative variables (age, gender, ASA score, BMI, comorbidities, and surgical history), operative variables (cause of reoperation, latency for reoperation, operative time, surgery performed, and cause of conversion), and post-operative variables (intestinal transit, hospital days, ICU days, medical complications, surgical site infection, evisceration, transfusion and 30-day mortality), were compared between groups. Results: There were no significant differences between groups among the pre-operative and operative variables. In terms of post-operative variables, the laparoscopic reoperation group, had fewer hospital days $(p=0.012)$, fewer ICU days $(p=0.001)$, and faster intestinal transit regarding gas, stool and return to solid diet $(p=0.008$, $p=0.029$ and $p=0.030$, respectively). However, there were no significant differences in surgical site infection, evisceration, medical complications, transfusion, and mortality. Discussion and Conclusion: This study revealed better post-operative clinical course in the laparoscopic reoperation group, with shorter hospital and ICU stay, and reduced postoperative ileus, without increased morbidity or mortality. Laparoscopic reoperation for complications after elective laparoscopic colorectal surgery may therefore be the preferred approach.
\end{abstract}

Keywords: laparoscopic colorectal surgery; surgical complication; reoperation.

\section{Resumen}

Objetivo: Comparar tres abordajes quirúrgicos (abierto, laparoscópico y laparoscópico convertido) para el manejo de complicaciones posoperatorias en cirugía colorrectal electiva realizadas primariamente por vía laparoscópica. Materiales y Método: Este estudio de cohorte retrospectivo incluyó pacientes reoperados después de una cirugía colorrectal laparoscópica electiva, agrupándose según la vía de abordaje de reoperación: abierta (RVA), laparoscópica (RVL) y laparoscópica convertida (RVLC). Las variables estudiadas fueron: preoperatorias (edad, sexo, puntuación ASA, IMC, comorbilidades e historia quirúrgica); operatorias (causa de reoperación, latencia para reoperación, tiempo operatorio, cirugía realizada y causa de conversión); y posoperatorias (tránsito intestinal, días de hospitalización, días de UCI, complicaciones médicas, infección del sitio quirúrgico, evisceración, transfusión y mortalidad a los 30 días). Resultados: Sin diferencias significativas para las variables preoperatorias y operatorias. En cuanto a las variables posoperatorias, el grupo de reoperaciones por vía laparoscópica, tuvo menos días de hospitalización $(\mathrm{p}=0,012)$, menos días de UCI $(\mathrm{p}=0,001)$ y un tránsito intestinal más rápido para reaparición de gases, heces y retorno a dieta sólida $(\mathrm{p}=0,008, \mathrm{p}=0,029, \mathrm{p}=0,030$, respectivamente).
Unidad de Coloproctología. Clínica INDISA. ${ }^{2}$ Universidad Andrés Bello, Facultad de Medicina Campus Clínica Indisa. Santiago, Chile.

Recibido 2121-03-25 aceptado 2121-04-24

Correspondencia a: Dr. Gonzalo Campaña V. gonzalo.campana@indisa.cl 
No hubo diferencias significativas en la infección del sitio quirúrgico, la evisceración, las complicaciones médicas, la transfusión y la mortalidad. Discusión y Conclusión: Este estudio reveló una mejor evolución clínica posoperatoria en el grupo de reoperación laparoscópica, con menor estancia hospitalaria y en UCI, y reducción del íleo posoperatorio, sin aumento de la morbimortalidad. Por lo tanto, la reoperación laparoscópica en cirugía colorrectal podría ser el abordaje más adecuado en pacientes debidamente seleccionados.

Palabras clave: cirugía colorrectal laparoscópica; complicación quirúrgica; reoperación.

\section{Introducción}

La prevalencia descrita en la literatura nacional y extranjera relacionada con el riesgo de complicaciones posoperatorias en la cirugía colorrectal fluctúa entre el $3 \%$ a $19 \%$, con tasas de mortalidad ante la presencia de complicaciones que se eleva hasta un $22 \%{ }^{1}$. Dado lo anterior, se establece que la incidencia global de reoperaciones en cirugía colorrectal varía entre un $2 \%$ y $12 \%$, determinado principalmente por la fuga anastomótica $(\mathrm{FA})^{2,3}$.

La principal causa de reingreso al quirófano en pacientes operados de cirugía colorrectal es la FA, que clínicamente se puede presentar como una colección pericolónica adyacente a la anastomosis hasta una peritonitis difusa, dependiendo del grado de dehiscencia anastomótica asociada ${ }^{3,4}$. El hemoperitoneo, lesiones inadvertidas y obstrucciones intestinales son otros de los diagnósticos más frecuentes a la hora de decidir una reintervención quirúrgica no planificada ${ }^{3,5,6}$.

La llegada de la cirugía laparoscópica ofrece las ventajas de la cirugía mínimamente invasiva en las patologías quirúrgicas colorrectales ${ }^{7}$, la que actualmente se considera como la vía de acceso más recomendada ${ }^{8,9}$, sin embargo, esta vía no está exenta de complicaciones existiendo siempre el riesgo de reintervenir ${ }^{7,10}$.

Publicaciones recientes sobre el manejo quirúrgico de complicaciones posteriores a las cirugías laparoscópicas más frecuentes, tales como la colecistectomía y cirugías bariátricas ${ }^{8}$, proponen el abordaje laparoscópico como la vía de elección para la reintervención, pudiendo resolver la complicación manteniendo todas las ventajas del abordaje mínimamente invasivo ${ }^{12}$. Sin embargo, aún es cuestionable esta conducta en las complicaciones posteriores a una cirugía laparoscópica colorrectal, ya que históricamente la preferencia ha sido la reintervención con laparotomía ${ }^{13}$.

Dentro de las desventajas del manejo laparoscópico de las complicaciones después de una cirugía colorrectal primariamente realizada por esta vía, se describen las dificultades técnicas tales como la falta de visibilidad por la distensión de las asas intestinales, dificultad para encontrar el punto de sangrado, la fragilidad del tejido para la manipulación con el instrumental laparoscópico, un mayor tiempo operatorio y la falta de experiencia del equipo quirúrgico ${ }^{5,14-16}$, siendo éstas las causas más frecuentes para la conversión a cirugía abierta $^{13,17,18}$. Por otro lado, considerar el estado clínico de los pacientes en un contexto séptico posoperatorio condiciona al cirujano en la elección prefiriendo el abordaje abierto ${ }^{19}$. Sin embargo, reportes sobre el manejo laparoscópico de la peritonitis difusa, proponen la vía laparoscópica como una alternativa segura con una menor tasa de infecciones del sitio operatorio, menor tiempo de hospitalización posoperatoria, reinserción laboral precoz, menos dolor posoperatorio y una menor tasa de hernias incisionales, entre otras ventajas $^{20-22}$.

La cantidad y la calidad de las publicaciones relacionadas es escasa, no existiendo literatura chilena al respecto.

El objetivo de este estudio es comparar los tres tipos de abordajes, abierto, laparoscópico y laparoscópico convertido, junto a su evolución clínica para el manejo de complicaciones quirúrgicas intraabdominales posterior a una cirugía laparoscópica colorrectal electiva.

\section{Materiales y Método}

Se realizó un estudio de cohortes con un análisis retrospectivo de los datos cuya muestra incluye a todos los pacientes con cirugía laparoscópica colorrectal electiva que requirieron reoperación debido a una complicación quirúrgica intraabdominal.

Los pacientes se agruparon según la vía de abordaje de la reoperación en reoperados por vía laparoscópica (RVL), reoperados por laparoscopía que requirieron de conversión a cirugía abierta (RVLC) y en reoperados por vía abierta (RVA). 
Se utilizaron datos provenientes de una base de datos prospectiva perteneciente al equipo de cirugía colorrectal de Clínica INDISA, entre los años 2004 y 2019.

Los criterios de exclusión fueron haber cursado con una conversión a cirugía abierta en la cirugía laparoscópica inicial, complicaciones manejadas con drenaje percutáneo, colecciones de pared abdominal y falta de seguimiento para la recolección de datos.

El análisis comparativo de las características clínico-demográficas basales previas a la reintervención de cada grupo consideró la edad, sexo, score ASA, IMC, comorbilidades, cirugías abdominales previas, diagnóstico en la primera intervención, tipo de cirugía realizada inicialmente, estado clínico previo a la reintervención determinado por la presencia de sepsis posoperatoria.

Se define como sepsis posoperatoria, el cuadro clínico secundario a un procedimiento quirúrgico que cursa con un SIRS (síndrome de respuesta inflamatoria sistémica) originado por un proceso infeccioso dentro de los 14 días después de un procedimiento quirúrgico mayor ${ }^{15}$.

Para medir la sepsis posoperatoria se utilizó el score de Quick-Sofa recomendado en la guía clínica 2016 de The National Institute of Health and Care Excellence (NICE) en Reino Unido, el que considera 3 aspectos clínicos relacionados con el riesgo de sepsis, a) frecuencia respiratoria $>22$ por minuto, b) estado mental alterado (Glasgow $<15$ ) y c) presión sistólica $<100 \mathrm{mmHg}$. Cada ítem con una puntuación de 1; con un valor total de 2 o más se consideró como sepsis en curso $^{23}$.

Los hallazgos quirúrgicos evaluados en las reoperaciones consideraron las causas de reintervención, latencia para la reoperación, tiempo operatorio, cirugía realizada y causas de conversión (grupo RVLC).

En la evolución clínica posoperatoria para los 3 grupos, se comparó el tránsito intestinal (gases, deposiciones, realimentación con sólidos), días de hospitalización, requerimientos de unidad de paciente crítico (UPC), complicaciones médicas (cardíacas, respiratorias, renales, infecciosas), infección del sitio operatorio (ISO), evisceración, transfusiones y mortalidad hasta los 30 días posoperatorios de la segunda cirugía.

\section{Análisis estadístico}

Se utilizó la prueba Shapiro Wilk para evaluar la normalidad de la muestra, Chi cuadrado de Pearson y el test exacto de Fisher para evaluar la asociación entre variables categóricas. La comparación de promedios de variables continuas entre dos grupos en estudio se realizó mediante la prueba de Kruskal
Wallis por su distribución anormal. Significación estadística fue evaluada a un nivel de 5\%.

\section{Aspectos éticos}

Los datos fueron extraídos con anonimización de la información, enviándola al Comité Ético Científico de la Universidad Andrés Bello, cumpliendo con las declaraciones Helsinki ${ }^{24}$ y Taipei ${ }^{25}$.

\section{Resultados}

De un total de 616 cirugías colorrectales laparoscópicas electivas, $56(9,09 \%)$ requirieron de una segunda intervención quirúrgica debido a una complicación intraabdominal; se excluyeron 17 pacientes: 11 convertidos en la primera cirugía, 2 colecciones de pared abdominal, 3 drenajes de colección intraabdominal percutáneo y 1 sin seguimiento. Considerando finalmente a 39 pacientes, 14 (36\%) reoperados completamente vía laparoscópica (grupo RVL), 17 casos (44\%) reoperados vía abierta (grupo RVA) y 8 pacientes $(21 \%)$ reoperados inicialmente vía laparoscópica que requirieron de conversión cirugía abierta (grupo RVLC).

En la Tabla 1 se observan las características clínico-demográficas de los tres grupos, no mostrando diferencias estadísticamente significativas.

Al evaluar los hallazgos intraoperatorios en las recirugías, no se encontraron diferencias significativas en la comparación de los grupos. La tasa de conversiones a cirugía abierta fue de un $36 \%(8 / 22)$, siendo las dificultades técnicas la principal causa $(62,5 \% ; 5 / 8)$ (Tabla 2).

La vía de abordaje en las reoperaciones posterior a las sigmoidectomías fue la RVL, colectomías derechas la RVA y resección de recto bajas la RVLC, aunque sin significancia estadística.

El 46\% los casos (18 pacientes) cursaron con una sepsis posoperatoria; el $50 \%$ de estos casos fueron reintervenidos por vía abierta y el $50 \%$ restante se intentó por vía laparoscópica, sin embargo, el 44,4\% (4/9) se convirtieron. La tasa total de filtración fue de un $6,3 \%$, siendo la principal causa de reoperación en todos los grupos. Hubo aproximadamente 2 días más de latencia para realizar la reintervención en el grupo RVL comparado con los otros grupos.

El tratamiento quirúrgico más utilizado (43\%) en el grupo RVL fue la ostomía en asa proximal con sutura de refuerzo sin deshacer la anastomosis (4 ileostomías y 2 colostomías). En los demás grupos fue la ostomía terminal el tratamiento más utilizado, con un $41 \%$ para RVA (3 ileostomías y 4 Hartmann) y $50 \%$ para RVLC (1 ileostomía y 3 Hartmann). 
Tabla 1. Características demográficas

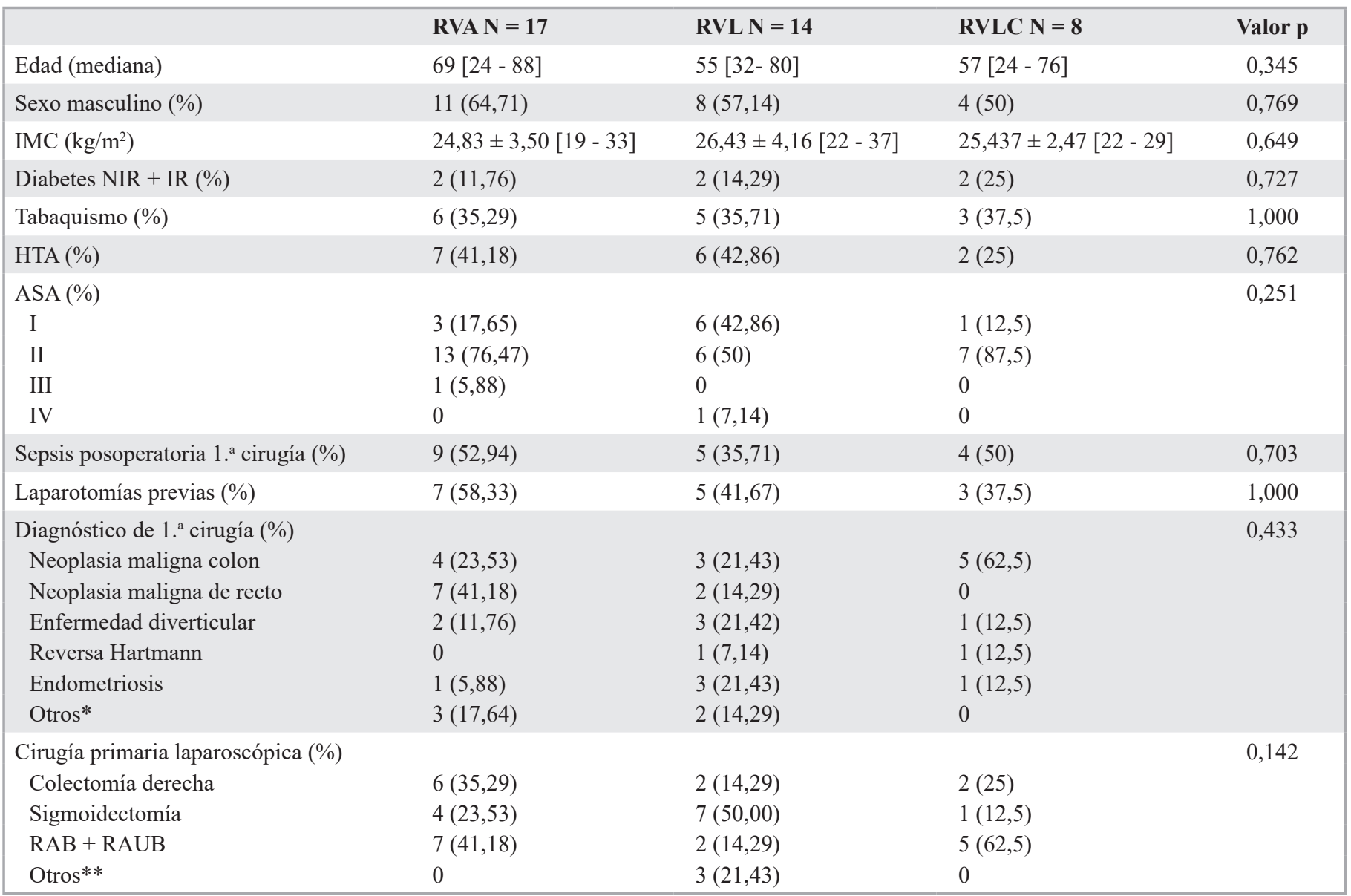

*Inercia colónica, lipomas de sigmoides. **Reversa Hartmann (1), Colectomía segmentaria (1), Colectomía total (1).

El tiempo operatorio de las reoperaciones fue similar en los tres grupos $(\mathrm{p}=0,861)$ (Tabla 2).

La tasa general de conversión del grupo RVL fue un $36,4 \%(8 / 22)$, sin embargo, en el grupo portador de sepsis fue mayor $(44,4 \%)$ (ver si ese $80 \%$ es verdad). Los principales motivos fueron la dificultad técnica (falta de visibilidad secundaria a la dilatación de las asas intestinales, dificultad para encontrar el punto de sangrado y fragilidad del tejido) y lesiones incidentales (Tabla 2).

La evolución posoperatoria más favorable para el grupo RVL al presentar un menor tiempo de hospitalización ( $p=0,012)$, menor tiempo de estadía en UPC $(\mathrm{p}=0,001)$ y mayor velocidad en recuperación del tránsito intestinal en expulsión de gases, deposiciones e ingesta de sólidos $(p=0,008, p=0,029$ y $\mathrm{p}=0,030$, respectivamente).

La ISO, evisceraciones, complicaciones médicas, transfusiones y mortalidad fueron más frecuentes en los grupos RLVC y RVA, aunque sin significancia estadística.

La mortalidad fue levemente superior en el grupo de pacientes RVA, aunque sin significancia estadística, siendo en todos estos casos originadas por complicaciones médicas (cardiacas y respiratorias) (Tabla 3).

\section{Discusión}

El enfrentamiento laparoscópico en las complicaciones quirúrgicas posteriores a una cirugía colorrectal inicialmente realizada por la misma vía está siendo respaldado en forma progresiva por evidencia que demuestra los beneficios de esta técnica por sobre el abordaje abierto convencional $2^{2,10,16}$. 
Tabla 2. Hallazgos intraoperatorios en recirugía

\begin{tabular}{|lllll|}
\hline & RVA N = 17 & RVL N = 14 & RVLC N = 8 & Valor p \\
Latencia para la reoperación (media, días) & $4,94 \pm 2,68[1-9]$ & $6,14 \pm 3,03[2-12]$ & $4,88 \pm 2,36[3-9]$ & 0,434 \\
Tiempo operatorio reoperación (media, minutos) & $140 \pm 39,31[56-234]$ & $144 \pm 53,66[74-254]$ & $152 \pm 31,38[126-223]$ & 0,861 \\
Causa de reoperación (\%) & & & & 0,557 \\
Fuga anastomótica & $11(64,71)$ & $9(64,29)$ & $6(75)$ & $2(25)$ \\
Hemoperitoneo & $2(11,76)$ & $2(14,29)$ & 0 \\
Obstrucción intestinal & $4(23,53)$ & $1(7,14)$ & 0 \\
Lesiones incidentales & 0 & $2(14,29)$ & $3(37,5)$ \\
Tratamiento en la reoperación (\%) & & & $1 / 2$ & 0,743 \\
Ostomía en asa & $3(17,65)$ & $6(42,86)$ & $4(50)$ \\
Ileostomía / colostomía & $2 / 2$ & $4 / 2$ & $1 / 3$ \\
Ostomía terminal & $7(41,18)$ & $4(28,57)$ & 0 \\
Ileostomía / Hartamnn & $3 / 4$ & $1 / 3$ & 0 \\
Refuerzo anastomosis + ostomía de protección & $1(5,88)$ & $1(7,14)$ & 0 \\
Aseo quirúrgico + drenajes & $2(11,76)$ & $2(14,29)$ & $1(12,5)$ \\
Adherenciolisis & $1(5,88)$ & $1(7,14)$ & $8 / 22(36,36)$ \\
Reconfección anastomosis sin ostomías & $3(17,65)$ & 0 & $5 / 8(62,5)$ \\
Conversión de relaparoscopía (\%) & - & - & $2 / 8(25)$ \\
Dificultad técnica & - & - & $1 / 8(12,5)$ \\
Lesión incidental de asa intestinal & - & - & - \\
Lesión incidental vascular & - & - & - \\
\hline
\end{tabular}

Tabla 3. Complicaciones y evolución posreoperación

\begin{tabular}{|lllll|}
\hline & RVA N = 17 & RVL N = 14 & RVLC N = 8 & Valor p \\
Días de hospitalización (media, DS) & $22,59 \pm 13,07[6-52]$ & $11,71 \pm 5,92[5-25]$ & $21,38 \pm 8,52[12-32]$ & 0,012 \\
Días UPC posoperatoria (media, DS) & $14,94 \pm 10,92[0-44]$ & $3,71 \pm 3,49[0-13]$ & $14,25 \pm 8,70[5-25]$ & 0,001 \\
Tránsito intestinal (media, días) & & & & \\
Gases & $5,14 \pm 3,79[2-16]$ & $2,69 \pm 0,72[2-4]$ & $3,88 \pm 0,991[3-5]$ & 0,008 \\
Deposiciones & $6,93 \pm 3,83[2-16]$ & $3,85 \pm 0,86[2-5]$ & $5,25 \pm 1,91[3-8]$ & 0,029 \\
Realimentación con sólidos & $11,33 \pm 5,43[6-22]$ & $8,38 \pm 5,82[4-12]$ & $9,00 \pm 4,75[6-20]$ & 0,030 \\
ISO (\%) & $5(29,41)$ & $3(21,43)$ & $3(37,5)$ & 0,737 \\
Evisceración (\%) & $1(5,88)$ & 0 & 0 & 1000 \\
Mortalidad a 30 días (\%) & $2(11,11)$ & $1(7,69)$ & 0 & 1000 \\
Complicaciones médicas (\%) & & & & 0,405 \\
Respiratorias & $29,41(5)$ & $14,29(2)$ & $3(37,5)$ & 0,485 \\
Cardiacas & $4(23,53)$ & $7,14(1)$ & $2(25)$ & 1000 \\
Renales & $1(5,88)$ & $7,14(1)$ & $4(50)$ & 0,499 \\
Transfusiones posreoperación (\%) & $11(64,7)$ & $6(42,85)$ & & \\
\hline
\end{tabular}


La morbilidad descrita asociada a la relaparoscopía en cirugía colorrectal es menor comparada con la relaparotomía ${ }^{5}$, sin embargo, sigue siendo la cirugía abierta la vía de abordaje actualmente recomendada en reintervenciones debido a la escasa cantidad y calidad de evidencia científica que avale a la vía laparoscópica $^{1,26}$. Esto a pesar de que se reconoce que la laparotomía como alternativa de tratamiento en las complicaciones de una cirugía colorrectal tiene un costo alto, aumentando la probabilidad de un resultado desfavorable en la evolución posoperatoria.

En el grupo RVL existió una mayor latencia para decidir la reoperación. Este grupo estuvo asociado a una menor proporción de sepsis posoperatoria, lo que hace suponer que el estado clínico pudiera jugar un rol importante a la hora de decidir la vía a utilizar para resolver la complicación quirúrgica.

Considerando que en este estudio un $36 \%$ de las complicaciones posteriores a una cirugía colorrectal laparoscópica electiva se lograron resolver completamente por relaparoscopía, se evidencia el rol relevante que tiene esta vía como herramienta terapéutica, más aún si logra una más rápida recuperación y sin agregar tiempo operatorio ni morbimortalidad.

Sin embargo, algunos estudios mencionan que la laparoscopía colorrectal convertida a cirugía abierta podría agregar mayor morbilidad que la laparotomía planteada desde un principio, en especial a los pacientes con cirugías oncológicas, ya que tendrían una menor sobrevida y un menor tiempo libre de enfermedad ${ }^{18,28}$.

La FA fue el motivo más frecuente de reoperación en los grupos, reproduciendo cifras similares en la literatura relacionada ${ }^{29}$.

Las ostomías en asa y sutura de refuerzo fueron las propuestas de tratamiento más frecuentes en el grupo de reintervenciones laparoscópicas, a diferencia del grupo de cirugía abierta en que el tratamiento más frecuente fue la ostomía terminal (operación de Hartmann). Esto pudiera estar relacionado con la sepsis ya descrita en el grupo abierto y el alto grado de dehiscencia anastomótica obligando a desmantelarla.

Por otro lado, la relaparoscopía implica un desafío técnico relevante, dado que un $36 \%$ (8 pacientes de un total de 22 hubo que convertirlas a cirugía abierta, siendo la mayoría $(62,5 \%)$ motivadas por dificultad técnica con imposibilidad de progresar en la cirugía.

Hubo 3 casos de conversión por lesiones incidentales laparoscópicas (intestinales y vasculares), confirmando que la relaparoscopía no está exenta de complicaciones y lesiones intraoperatorias, al igual que en la cirugía primaria ${ }^{30}$.
La evolución posoperatoria del grupo RVL fue favorable, requiriendo menos UPC posoperatoria, presentando una recuperación del tránsito intestinal precoz, determinando un menor tiempo de hospitalización y un retorno anticipado a las actividades habituales, ventajas ya reportadas sobre el abordaje laparoscópico en una reoperación ${ }^{18,31}$.

Con relación a la evolución posoperatoria de los pacientes convertidos (RVLC), no se observaron grandes diferencias al compararla con el grupo RVA, lo que traduce que la conversión a cirugía abierta no agregaría mayor morbimortalidad en estos pacientes. Al observar que la principal causa de conversión fue la dificultad técnica, sería interesante investigar en futuros estudios el intervalo de tiempo invertido en la cirugía para tomar la decisión de convertir, ya que es conocido que mientras mayor es el tiempo de exposición ante las dificultades técnicas de una laparoscopía, mayor es el riesgo de lesiones iatrogénicas y el tiempo quirúrgico total ${ }^{28}$, aumentando la morbilidad y mortalidad en estos pacientes.

Impresiona que los datos relacionados con ISO, evisceración y mortalidad requieren de un mayor número de pacientes para establecer conclusiones más robustas en el análisis de los 3 grupos.

Al evaluar la mortalidad a 30 días, la principal causa de muerte de los 3 pacientes registrados, fueron secundarias a complicaciones cardiorrespiratorias en pacientes mayores de 73 años que cursaron con cuadros sépticos secundarios a la FA.

Las limitaciones de este estudio son el hecho de ser retrospectivo, no aleatorizado, número muestral pequeño, necesitando mayor universo para darle peso estadístico a estos resultados.

Se puede distinguir que las ventajas de la recirugía realizada por vía laparoscópica no se aplican a los casos convertidos, dejando una puerta abierta a la investigación dirigiendo los esfuerzos en identificar los factores que colaboraron en la decisión de realizar directamente una cirugía abierta evitando una conversión, asumiendo que ésta tiene riesgos y podría tener costos mayores.

\section{Conclusiones}

Consideramos que las reoperaciones laparoscópicas motivadas por complicaciones posoperatorias de cirugías colorrectales primariamente realizadas por la misma vía, podría ser beneficiosa en pacientes debidamente seleccionados, ya que cursarán con una mejor evolución posoperatoria comparadas con las otras vías presentando un menor requerimiento de estadía en UPC, menos íleo posquirúrgico y una 
estadía hospitalaria más corta sin agregar mayor tasa de morbimortalidad.

\section{Responsabilidades éticas}

Protección de personas y animales. Los autores declaran que para esta investigación no se han realizado experimentos en seres humanos ni en animales.

Confidencialidad de los datos. Los autores declaran que en este artículo no aparecen datos de pacientes.

Conflictos de interés: no hay.

\section{Bibliografía}

1. Vennix S, Abegg R, Bakker OJ, Van Den Boezem PB, Brokelman WJA, Sietses C, et al. Surgical re-interventions following colorectal surgery: Open versus laparoscopic management of anastomotic leakage. J Laparoendosc Adv Surg Tech. 2013;23:739-44.

2. Amoruso M, D'Abbicco D, Notarnicola A, Angrisano A, Nacchiero M, Martino Bonomo G. [Early colorectal reoperation: risk factors]. Chir Ital [Internet]. [cited 2019 Nov 5];53(6):827-33. Available from: http://www.ncbi.nlm.nih.gov/ pubmed/11824058.

3. Bannura G, Cumsille MA, Barrera A, Contreras J, Melo C, Soto D, et al. Reoperaciones precoces en cirugía colorrectal. Análisis uni y multivariado de factores de riesgo. Rev Chil Cir. 2007;59:281-6.

4. Lee CM, Huh JW, Yun SH, Kim HC, Lee WY, Park YA, et al. Laparoscopic versus open reintervention for anastomotic leakage following minimally invasive colorectal surgery. Surg Endosc. 2015;29:931-6.

5. Rotholtz NA, Laporte M, Lencinas SM, Bun ME, Aued ML, Mezzadri NA. Is a laparoscopic approach useful for treating complications after primary laparoscopic colorectal surgery? Dis Colon Rectum 2009;52:275-9.

6. Chang KH, Bourke MG, Kavanagh DO, Neary PC, O'Riordan JM. A systematic review of the role of re-laparoscopy in the management of complications following laparoscopic colorectal surgery. Surgeon [Internet]. 2016;14(5):287-93. Available from: http://dx.doi.org/10.1016/j. surge.2015.12.003.

7. Cuccurullo D, Pirozzi F, Sciuto A, Bracale U, La Barbera C, Galante F, et al. Relaparoscopy for management of postoperative complications following colorectal surgery: ten years experience in a single center. Surg Endosc. 2015;29:1795-803.

8. Zhou S, Zhu H, Li Z, Ying X, Xu M. Safety of laparoscopic resection for colorectal cancer in patients with liver cirrhosis: A retrospective cohort study. Int J Surg. 2018;55:110-6.

9. Vignali A, Braga M, Zuliani W, Frasson M, Radaelli G, Di Carlo V. Laparoscopic Colorectal Surgery Modifies Risk Factors for Postoperative Morbidity. Dis Colon Rectum [Internet]. 2004 Oct [cited 2019 Aug 12];47(10):1686-93. Available from: https://insights.ovid.com/crossref? an $=00003453-200447100-00018$.

10. Boyce SA, Harris C, Stevenson A, Lumley J, Clark D. Management of Low Colorectal Anastomotic Leakage in the Laparoscopic Era: More Than a Decade of Experience. Dis Colon Rectum 2017;60:807-14

11. Pohl D, Schmutz G, Plitzko G, Kröll D, Nett P, Borbély Y. Perforated duodenal ulcers after Roux-Y Gastric Bypass. Am J Emerg Med. 2018;36:1525. e1-1525.e3.

12. Frantzides CT, Alexander B, Frantzides AT. Laparoscopic Revision of Failed Bariatric Procedures. JSLS J Soc Laparoendosc Surg. 2019;23:e2018.00074.

13. Ibáñez N, Abrisqueta J, Luján J, Sánchez P, Soriano MT, Arevalo-Pérez J, et al. Reintervención tras complicaciones en cirugía laparoscópica colorrectal. ¿Aporta ventajas el abordaje laparoscópico? Cir Esp. 2018;96:109-16.

14. Agresta F, Mazzarolo G, Ciardo LF, Bedin N. The laparoscopic approach in abdominal emergencies: Has the attitude changed? A single-center review of a 15year experience. In: Surgical Endoscopy and Other Interventional Techniques. Springer; 2008. p. 1255-62.

15. Yarmuch J, Mayanz S, Romero C.
Sepsis severa y cirugía. Rev Chil Cir. 2015;67:79-87.

16. López KF, Suazo LC, Heine TC, Abedrapo MM, Avendaño HR, Germain PF, et al. Cirugía laparoscópica colorrectal en Chile. Rev Chil Cir. 2011;63:479-84

17. Van der Pool A, Rawlings A, SánchezGuillén L, Kuiper S, Negoi I, Buchs N, et al. The impact of conversion on the risk of major complication following laparoscopic colonic surgery: an international, multicentre prospective audit. Color Dis [Internet]. 2018 Sep 1 [cited 2020 Mar 25];20:69-89. Available from: http://www.ncbi.nlm.nih.gov/ pubmed/30255643.

18. Belizon A, Sardinha CT, Sher ME. Converted laparoscopic colectomy: What are the consequences? Surg Endosc Other Interv Tech. 2006;20:947-51.

19. Linder MM, Wacha H, Feldmann U, Wesch G, Streifensand RA, Gundlach E. [The Mannheim peritonitis index. An instrument for the intraoperative prognosis of peritonitis]. Chirurg [Internet]. 1987 Feb [cited 2020 Mar 11];58(2):84-92. Available from: http://www.ncbi.nlm.nih. gov/pubmed $/ 3568820$.

20. Gomes CA, Sartelli M, Podda M, Di Saverio S, Coccolini F, Segovia-Lohse HA, et al. Laparoscopic versus open approach for diffuse peritonitis from appendicitis ethiology: a subgroup analysis from the Physiological parameters for Prognosis in Abdominal Sepsis (PIPAS) study. Updates Surg [Internet]. 2020 Mar [cited 2020 Mar 19];72(1):185-91. Available from: http://www.ncbi.nlm.nih.gov/ pubmed/32077062.

21. Feingold D, Steele SR, Lee S, Kaiser A, Boushey R, Buie WD, et al. Practice parameters for the treatment of sigmoid diverticulitis. Dis Colon Rectum [Internet]. 2014 Mar [cited 


\section{ARTÍ́CULO ORIGINAL}

2020 Mar 11];57(3):284-94. Available from: http://www.ncbi.nlm.nih.gov/ pubmed/24509449.

22. Tengberg LT, Cihoric M, Foss NB, BayNielsen M, Gögenur I, Henriksen R, et al. Complications after emergency laparotomy beyond the immediate postoperative period - a retrospective, observational cohort study of 1139 patients. Anaesthesia [Internet]. 2017 Mar 1 [cited 2020 Mar 11];72(3):309-16. Available from: http://www.ncbi.nlm.nih. gov/pubmed/27809332.

23. Herrod PJJ, Cox M, Keevil H, Smith KJE, Lund JN. NICE guidance on sepsis is of limited value in postoperative colorectal patients: The scores that cry "wolf!" Ann R Coll Surg Engl. 2018;100:275-8.

24. World Medical Association. World Medical Association Declaration of Helsinki. Ethical Principles for Medical Research Involving Human Subjets. Jama.2013;310:2191-4.

25. Asociación Médica Mundial. Declaración de Taipei sobre Consideraciones Éticas de las bases de datos de salud y Biobancos.
Asociación médica mundial. FerneyVoltaire, Francia [citado el 10 de agosto de 2017]. Disponible desde: https://www. wma.net/es/policies-post/declaracionde-laamm-sobre-las-consideracioneseticasde-las-bases-de-datos-de-salud-ylosbiobancos/.

26. Lee CM, Huh JW, Yun SH, Kim HC, Lee WY, Park YA, et al. Laparoscopic versus open reintervention for anastomotic leakage following minimally invasive colorectal surgery. Surg Endosc. 2015;29:931-6.

27. Watson AJM, Hudson J, Wood J, Kilonzo M, Brown SR, McDonald A, et al. Comparison of stapled haemorrhoidopexy with traditional excisional surgery for haemorrhoidal disease (eTHoS): a pragmatic, multicentre, randomised controlled trial. Lancet [Internet]. 2016;388(10058):2375-85. Available from: http://dx.doi.org/10.1016/S01406736(16)31803-7

28. Duraes L de C, Steele SR, Camargo MGM de, Gorgun E, Kalady MF, Valente M, et al. Conversion to open from laparoscopic colon resection is a marker for worse oncologic outcomes in colon cancer. Am J Surg [Internet]. 2019 Mar 1 [cited 2020 Mar 25];217(3):491-5. Available from: http://www.ncbi.nlm.nih.gov/ pubmed/30392676.

29. Chambers WM, Mortensen NJMC. Postoperative leakage and abscess formation after colorectal surgery. Best Pract Res Clin Gastroenterol. 2004;18:865-80.

30. Sarli L, Iusco DR, Regina G, Sansebastiano G, Ferro M, Veronesi L, et al. Predicting conversion to open surgery in laparoscopic left hemicolectomy. Surg Laparosc Endosc Percutaneous Tech. 2006;16:212-6.

31. Li J, Guo H, Guan XD, Cai CN, Yang LK, Li YC, et al. The Impact of Laparoscopic Converted to Open Colectomy on Short-Term and Oncologic Outcomes for Colon Cancer. J Gastrointest Surg [Internet]. 2015 Feb [cited 2020 Mar 26];19(2):335-43. Available from: http://www.ncbi.nlm.nih. gov/pubmed/25355006. 